\title{
Determination of recombination coefficients for nanocrystalline silicon embedded in hydrogenated amorphous silicon
}

\author{
Wei He ${ }^{1}$, Ammar ZaKaR ${ }^{1}$, Thomas Roger ${ }^{1}$, Igor V.YURKeVich ${ }^{2}$, ANd ANdRe KAPLAN ${ }^{1 .^{*}}$ \\ ${ }^{1}$ University of Birmingham, School of Physics and Astronomy, Birmingham, UK, B15 2TT \\ ${ }^{2}$ Aston University, Nonlinearity and Complexity Research Group, Birmingham, UK, B4 7ET \\ *Corresponding author: a.kaplan.1@bham.ac.uk
}

Compiled July 29, 2015

The spectroscopic pump-probe reflectance method was used to investigate recombination dynamics in samples of nano crystalline silicon embedded in a matrix of hydrogenated amorphous silicon. We found that the dynamics can be described by a rate equation including linear and quadratic terms corresponding to recombination processes associated with impurities and impurityassisted Auger ionisation, respectively. We determined the values of the recombination coefficients using the initial concentrations method. We report the coefficients of $1.5 \times 10^{11} \mathrm{sec}^{-1}$ and $1.1 \times 10^{-10} \mathrm{~cm}^{3} \mathrm{sec}^{-1}$ for the impurity-assisted recombination and Auger ionization, respectively. (๑) 2015 Optical Society of America

OCIS codes: (160.0160) Materials; (040.6040) Silicon; (310.0310) Thin films; (320.0320) Ultrafast optics

http://dx.doi.org/10.1364/ao.XX.XXXXXX

In recent decades, nanocrytalline silicon embedded in hydrogenated amorphous silicon (nc-Si:H) has attracted considerable attention, because of its capacity to improve and extend the capabilities of traditional silicon electronic, optoelectronic, and photonic devices[1]. For example, reports exist on the development of solar cells[2, 3], memory devices[4], and transistors [5]. On the other hand, it has been determined that parasitic effects such as free-carrier absorption and fast non-radiative recombination occur in nanocrystalline Si embedded in a matrix material; these effects severely limit the device efficiency[6]. It has been shown that the recombination and relaxation processes, especially at high carrier concentration, are enhanced and occur at a faster rate[7-9] than in bulk silicon[11, 12]. Further, the main recombination process in highly doped or injected bulk silicon, with a carrier concentration of $10^{18} \mathrm{~cm}^{-3}$ or higher, was quantified and demonstrated to be of Auger type[13, 14]. It has also been demonstrated that the recombination time in silicon has a predominant quadratic dependence on the carrier concentration and proceeds with an Auger coefficient of approximately $10^{-31} \mathrm{~cm}^{6} \mathrm{~s}^{-1}$ [13]. For comparison, the carrier population decay in amorphous hydrogenated silicon is a more complex bimodal process consisting of fast and slow components of different origins. The former corresponds to electron-hole recombination and trapping, while the latter is related to intraband migration of the trapped charges and their recombination with thermally emitted free carriers, with corresponding rate constants of $2.3 \times 10^{-8}$ and $6 \times 10^{-9} \mathrm{~cm}^{3} \mathrm{~s}^{-1}[15]$, respectively.

In this work we attempted to quantify the recombination coefficients in samples comprising nanocrystalline silicon embedded in a hydrogenated amorphous silicon matrix. Specifically, we investigated the carrier population decay dynamics using femtosecond (fs) pump-probe spectroscopic reflectance measurements. The decay process dynamics for the initial photo-excited carrier concentration were investigated over a timespan of 20 ps in the range of initial concentration between $2 \times 10^{19}$ and $9 \times 10^{19} \mathrm{~cm}^{-3}$. We found that, in this regime and timespan, the decay is best described by a kinetic rate model involving linear and quadratic terms corresponding to the recombination of a free carrier with a deep-level traps, and band-to-band recombination simultaneous with neutral impurity ionisation (Auger ionisation), respectively. To quantify the related coefficients we analysed the carrier decay curves by use of the initial carrier concentration method which is widely used in chemistry to determine reaction rate[16, 17]. We found that the carrierimpurity recombination occurs with a rate of $1.5 \times 10^{11} \mathrm{sec}^{-1}$, while the coefficient of Auger ionization was determined to be $1.1 \times 10^{-10} \mathrm{~cm}^{3} \mathrm{sec}^{-1}$.

For the measurements we used a Coherent laser system incorporated in the fs pump-probe setup, which can deliver $50-f_{s}$ pulses with a epetition rate of $1 \mathrm{kHz}$. The laser pulse had an almost Gaussian-shaped spectrum centered around $795 \mathrm{~nm}$ with a bandwidth of $60 \mathrm{~nm}$. A beam splitter was used to split the laser into two parts, namely, the pump and probe beams. The power ratio between the pump and probe was more than 100:1. A retroreflector delay stage was used to control the difference between the arrival times of the pump and probe pulses. A combination of a half wave plate and Brewster angle reflection from a glass block was used to adjust the pump fluence in the range between $0.14 \mathrm{~mJ} / \mathrm{cm}^{2}$ to $2.29 \mathrm{~mJ} / \mathrm{cm}^{2}$. The incident angles of the probe and pump beams were set to $70^{\circ}$ and $50^{\circ}$, respectively. A non-collinear configuration was used to avoid the interference 
in time domain. The polarization of the probe beam was adjusted to yield equal contributions of $s-$ and $p$-components, while the pump beam was orthogonally polarized with respect to the probe beam to prevent interference. The diameters of the beam spots were $300 \mu \mathrm{m}$ and $50 \mu \mathrm{m}$ for the pump and probe, respectively. The spatial overlap of the beams on the surface of the samples was imaged and adjusted with the help of a microscope. The temporary overlap was confirmed by the generation of a second harmonic through a thin barium borate (BBO) crystal. The reflected probe beam was wavelength-analyzed by an Ocean Optics QE65 Pro spectrometer. The detected data is presented here as $\Delta R / R_{0}$, where $R_{0}$ is the reflectance of the sample without pumping and $\Delta R$ is the absolute change of the reflectance induced by the pump excitation. More details of the pump-probe setup and measurements can be found elsewhere[8-10].

The nc-Si:H samples used in this study were provided by Sirica DC (Israel). The samples consisted of a $\sim 480 \mathrm{~nm}$ thick film of nanocrystalline silicon embedded in a hydrogenated amorphous silicon film grown on a $\sim 200 \mathrm{~nm}$ silicon dioxide layer on a crystalline silicon substrate.The specifics of the film growth method are similar to those described elsewhere[18]. We characterized the samples by means of Raman spectroscopy[19] and X-ray diffraction analysis[20-22]. This analysis, which has been published elsewhere[23], suggests that approximately $35 \%$ of the volume fraction is occupied by silicon nanocrystals with a mean diameter of $\langle a\rangle=6 \mathrm{~nm}$, having a rather broad size distribution extending between 3 and $10 \mathrm{~nm}$. A commercial ellipsometer was used to characterize the effective complex dielectric function, $\epsilon_{\text {eff }}^{0}$, of the film which drops monotonically from 12.25 to 11.8 and from 0.05 to 0.02 , for the real and imaginary parts, respectively, for the spectrum region spanning between $760 \mathrm{~nm}$ and $820 \mathrm{~nm}[9]$.

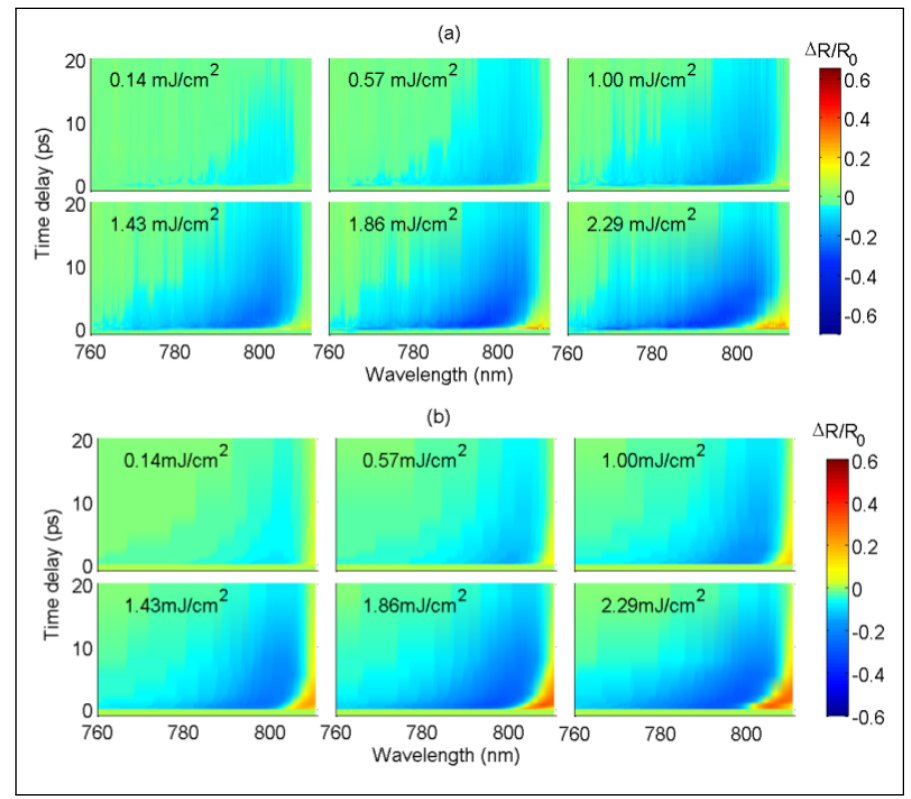

Fig. 1. Spectroscopic pump-probe reflectance change, $\Delta R / R_{0}$, measured for different pump fluencies (top). Theoretical simulation (bottom).

The top panel of Figure 1 shows the typical experimental pump-probe spectra of the reflectance change taken at different pump fluencies. We note that the measurements performed on other samples from the batch reproduced well the results shown here. The variance of the measured reflectance change was within the limits of a few percents. The reflectance primarily undergoes a negative change near the zero delay time at lower fluencies, while, at higher fluencies a Fabry-Perot fringe is observed at longer wavelengths. Spectra with similar Fabry-Perrot fringe features have been observed and explained previously for thin silicon film samples on a sapphire substrate[24]. Here we briefly note that the fringe originates from the interference effects of the probe beam reflected from the sample interfaces. The reflectance change recovers back to the values close to the background level within 20 picoseconds. These spectra were used for a model simulation procedure, which allowed the excited carrier concentration as a function of time to be retrieved. The details of the simulation are described in our previous studies[9, 23]. Here we briefly note that the simulation is based on the optical model obtained with the ellipsometry and the reflectance measurements of the samples at their ground state, i.e. without pumping. To account for the excitation induced by the pump, we added a Drude contribution to the effective dielectric function, $\epsilon_{e f f}^{0}$, of the top layer of nc-Si:H[8]. The short relaxation time[9, 23] (short with respect to the characteristic time scale of all other mechanisms and external perturbations) means that one can use adiabatic approximation which is dc conductivity with slowly changing parameters. That is why one can use simple Drude formula with adiabatically (with respect to other relaxation times) changing parameters. Thus, the updated effective dielectric function is expressed as the following:

$$
\epsilon_{e f f}=\epsilon_{e f f}^{0}-\frac{\omega_{p}^{2}}{\omega^{2}+i \Gamma \omega},
$$

where $\omega$ and $\omega_{p}$ are the probing and plasma frequencies, respectively, and $\Gamma$ is the scattering rate. The plasma frequency is given by

$$
\omega_{p}^{2}=\frac{e^{2} N_{e h}}{\epsilon_{0} m^{*} m_{e}},
$$

with $e, N_{e h}, m_{e}$ and $m^{*}$ representing the electron charge, carrier concentration, free electron mass, and effective mass, respectively. From Eq. 1, we can see that the change induced by the pump to the dielectric function is related to the changes in $\omega_{p}$ and $\Gamma$. Thus, incorporating $\epsilon_{e f f}$, into the optical model, we can simulate $\Delta R(\lambda, t) / R_{0}$ spectra (where $\lambda$ and $t$ are wavelength and time, respectively) with two fitting variables, i.e., $\omega_{p}$, and $\Gamma$. The fitting procedure yields reasonably good confidence, because it simultaneously determines only two fitting parameters, $\omega_{p}$, and $\Gamma$, for each wavelength of the probe spectrum. Thus, we kept $\omega_{p}$, and $\Gamma$ fixed to solve Equation 1 for each wavelength with the resolution of the detector to determine the entire of the pump-probe reflectance. The obtained $\omega_{p}$ are used to calculate the excited carrier concentration $N_{e h}$ from the definition of the plasma frequency given above. In our calculations, we used $m^{*}=0.17$, which has been estimated previously[9]. The simulated pump-probe spectra of $\Delta R(\lambda, t) / R_{0}$ are shown in Figure $1 \mathrm{~b}$, and the corresponding fitting values of $N_{e h}(t)$ and $\Gamma(t)$ for the different pumping fluencies are shown in Figure 2. In this paper, we discuss the time-dependent behaviour of the carrier population, $N_{e h}(t)$, only, leaving $\Gamma(t)$ outside of the scope of our arguments. It can be seen that, as expected, the initial carrier population at the zero-delay increases as the fluence increases. However, it is also clear that the population decays faster as the initial carrier population increases, indicating nonlinearity of the decay process. 


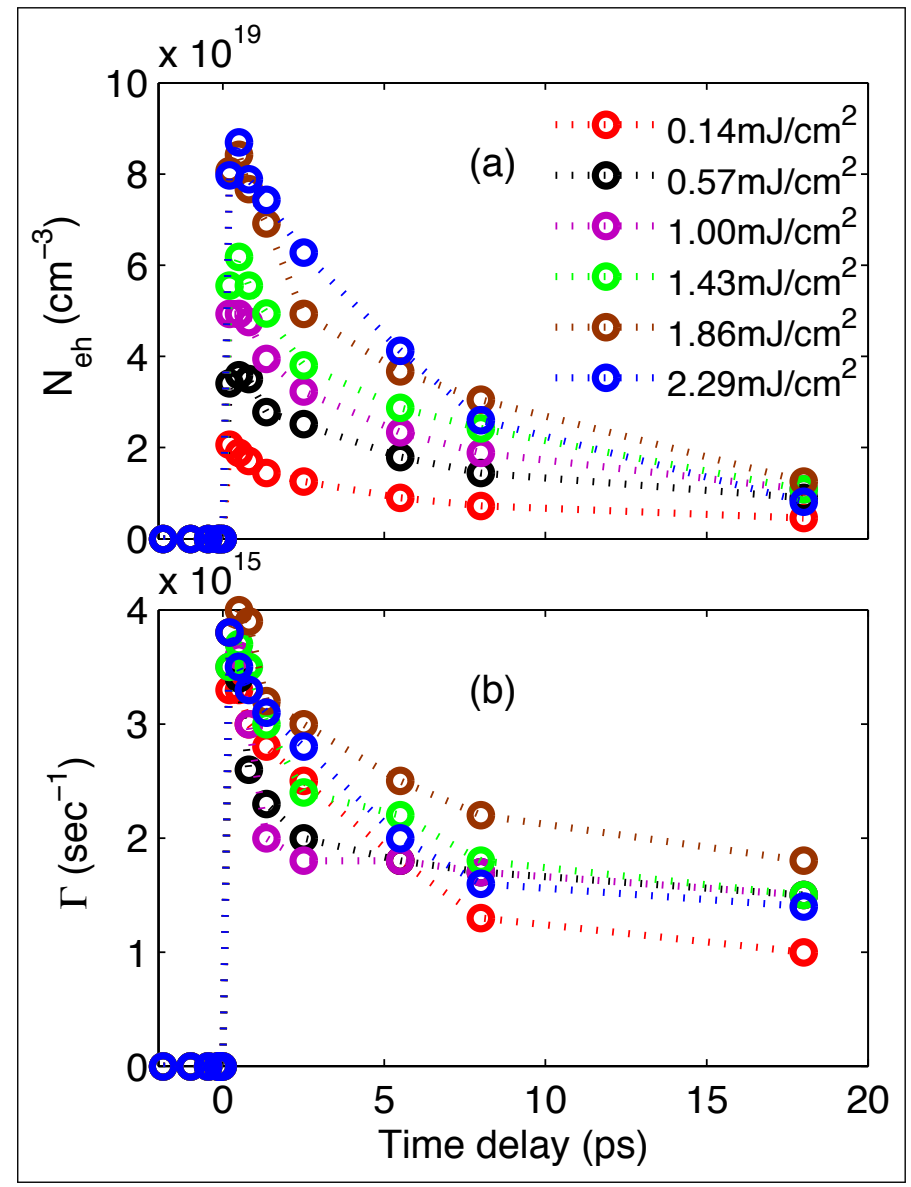

Fig. 2. (a) Charge carrier concentration, $N_{e h}$, and (b) the carrier scattering rate, $\Gamma$, as a functions of time obtained for different pump fluencies, obtained from the simulation of the experimental results shown in Figure 1.

The decay rate equation can be presented as the following[25]:

$$
-\frac{d N_{e h}(t)}{d t}=c N_{e h}(t)+b N_{e h}^{2}(t)
$$

The first linear term is a Shockley-Read-Hall (SRH) type recombination, which is associated with recombination via deep energy levels[26]. In this process the localized state not only absorbs energy, but also takes in differences in momentum. This process can be quite dominant in indirect band-gap semiconductors. The second, bimolecular quadratic term is associated with either band-to-band recombination (radiative or non-radiative), or the trap-assisted Auger ionization[27]. We can discard the radiative recombination, as no luminescence is detected for the investigated samples. On the other hand, the non-radiative bandto-band recombination process in silicon and silicon derivative materials occurs on much longer time scales than we observed here. For example, in silicon clusters the fastest rate rate of recombination is on microsecond time scale[28]. Therefore, our interpretation is related to the trap-assisted Auger process only. In this process, an electron-hole pair recombines, transferring liberated energy to a neutral impurity that is ionised with a release of a charge carrier. This carrier can be either a free electron or a hole. We note that higher terms are possible, but we found that a second order rate equation sufficies to describe the observed carrier decay dynamics. The parameters $c$ and $b$ are the recombination coefficients for the corresponding linear and quadratic terms, respectively. Unfortunately, these coefficients are not always easy to derive directly from an experimental decay curve because they might depend on other factors which change as a function of time, i.e. temperature, carrier diffusion rate and band-gap energy[29]. To avoid possible complications we analyzed our data using a method of initial concentrations.

The core of the method involves approximating the tangent to the concentration-time curve[16]. In our work, we determined the initial recombination rate, $\frac{d N_{e h}}{d t}$, from a linear fit over a time window of 2 picoseconds (ps) for different initial carrier concentrations. The fit is shown in Figure 3 on the top and middle panels. Then the obtained rates were plotted against the initial carrier concentration, $N_{e h}(0)$, and the coefficients $c$ and $b$ were retrieved from the second order polynomial fit. The polynomial fit is shown on the bottom panel of Figure 3. The best polynomial fit provided the values of $1.5 \times 10^{11} \mathrm{sec}^{-1}$ and $1.1 \times 10^{-10} \mathrm{~cm}^{3} \mathrm{sec}^{-1}$ for the coefficients $c$ and $b$, respectively.

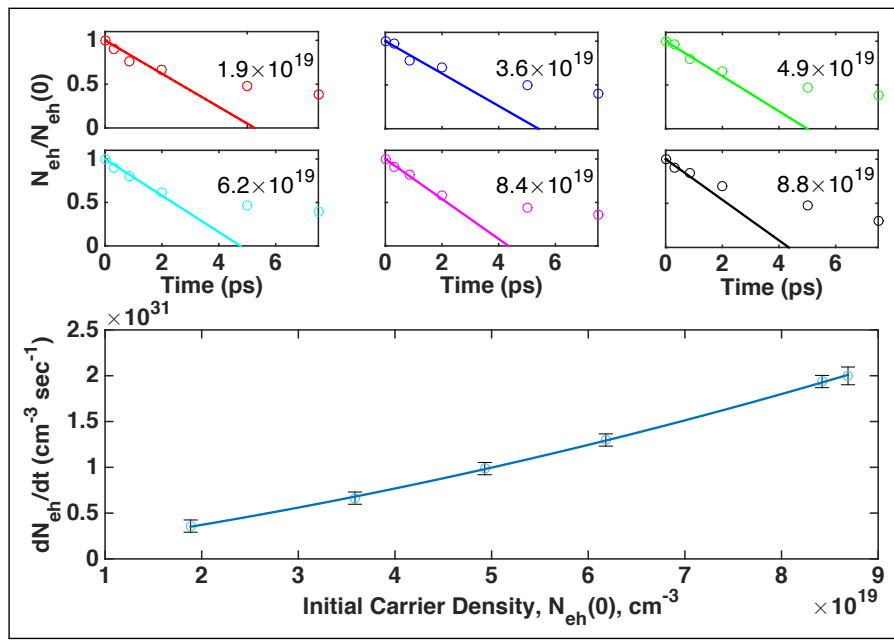

Fig. 3. Top and middle panels: The open circles represent the normalised carrier density as a function of time, each plot corresponds to initial carrier densities, $\mathrm{N}_{e h}(0)$, of 1.9, 3.6, 4.9, $6.2,8.4,8.8 \times 10^{19} \mathrm{~cm}^{-3}$, as indicated on the plots. The solid lines represent linear fits to determine recombination rates at initial times. Bottom panel: recombination rate as a function of the initial carrier concentration, $\mathrm{N}_{e h}(0)$. The open circles represent the experimentally determined rates from the data shown on the top and middle panels. The solid line depicts a fitting curve.

To test the validity of the approach we plotted the solution of Equation 3 given as the following:

$$
N(t)=\left[\left(\frac{1}{N_{e h}(0)}+\frac{b}{c}\right) e^{c t}-\frac{b}{c}\right]^{-1}
$$

together with the experimental decay curves on Figure 4. Overall, the experimental data is reasonably well described by Equation 4 over the first 10 ps and somewhat overestimate the decay for the last point of 20 ps, except for the highest initial concentration where agreement with the experiment is much better. The reason for the discrepancy at longer times is likely due to experimental error, because at 20 ps the detected signal is rather weak and the determination of the concentration is prone to the overestimation. 
Although the theoretical calculation of the recombination coefficients in this material has not yet been reported in the literature, and we can not compare the obtained experimental results to theoretical values, we can relate our findings to those published for similar material for matrix of amorphous silicon and bulk silicon. The majority of the works agree that, in amorphous silicon at higher pumping, the decay process is governed the non-radiative recombination with a quadratic term dominating the rate equation. These studies report values of the quadratic term lying in ranges similar to those we obtained in this work $[15,30,31]$. On the other hand, in bulk crystalline silicon the three-particle Auger recombination is the main recombination process at these carrier concentrations[13, 14, 35], in the contrast to our findings here. In addition, the coefficient responsible for the SRH recombination determined in this work is significantly higher than that for the bulk silicon, and, in fact, competes with the bimolecular recombination. The likely reason for a relative high rate of the SRH process is the high density of defects and corresponding in-gap states occupying the Urbach tail in the matrix material. Thus, after the excitation, an excited free carrier might recombine with its counterpart of the opposite polarity transferring the liberated energy to an impurity and ionising it. On the other hand, a nascent ionised impurity will capture another free carrier in transition between bands competing with the band-to band recombination.

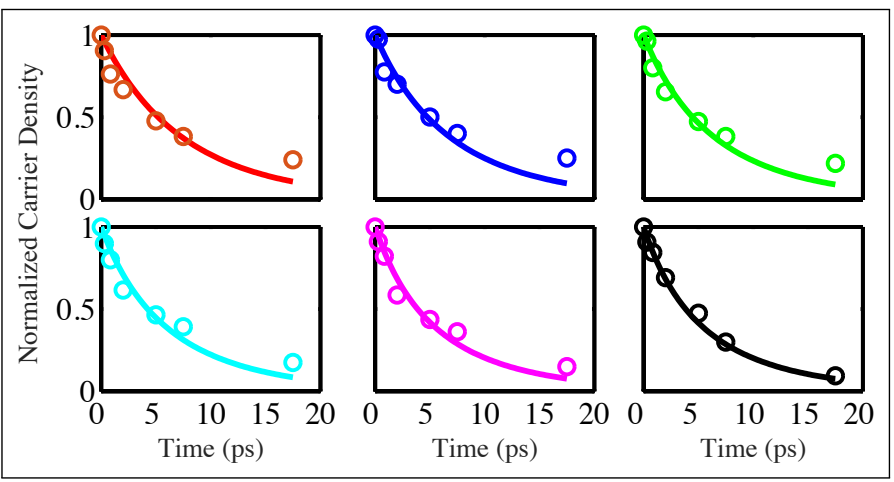

Fig. 4. The open circles represents the experimental normalised carrier density as a function of time. The data reproduced from Figure 3. The solid lines represent the calculated curves.

In summary, we measured the ultrafast time-resolved spectroscopic reflectance of the samples of nc-Si:H using the pumpprobe method. The obtained data were simulated with the aid of an optical model that incorporated the contribution of the complex Drude optical free-electron response. The simulation revealed a family of time-dependent carrier-density curves. These experimental findings were fitted against solutions to a recombination rate equation, and the recombination coefficients were obtained using the method of initial concentrations. These coefficients were related to the SRH and two-particle Auger ionization, and we found that the former proceeds much faster than in bulk crystalline silicon, while the later is of the same order of magnitude as the decay in amorphous silicon. The information provided on the recombination dynamics in this paper should help in the design of electronic and optoelectronic devices. The knowledge of the recombination coefficients in combinations with the diffusion coefficients allows to simulate fully the transport property of the material and make a correct decision about device configuration and expected performance.

\section{FUNDING INFORMATION}

The Engineering and Physical Research Council (EPSRC), EP/K503873/1; Defence Science and Technology Laboratory (Dstl)

\section{ACKNOWLEDGMENTS}

We thank D. Chekulaev for help with the experimental setup. We also thank Sirica DC for the provision of samples and assistance with their characterization.

\section{REFERENCES}

1. R. A. Street, Phys. Status Solidi A 166, 695-705 (1998).

2. A. Shah, P. Torres, R. Tscharner, N. Wyrsch, and H. Keppner, Science 285, 692-698 (1999).

3. P. S. Yeh, C.-W. Chen, B.-R. Yang, and L.-S. Hong, Appl. Opt. 53, 28472852 (2014).

4. S. Jung and J. Yi, Electron Device Letters, IEEE 31, 981-983 (2010).

5. C.-H. Lee, A. Sazonov, A. Nathan, and J. Robertson, Appl. Phys. Lett. 89, 252 (2006).

6. R. D. Kekatpure and M. L. Brongersma, Phys.Rev. A 78, 023829 (2008).

7. K. E. Myers, Q. Wang, and S. L. Dexheimer, Phys. Rev. B 64, 161309 (2001).

8. J. Barreto, T. Roger, and A. Kaplan, Appl. Phys. Lett. 100, 241906 (2012).

9. T. W. Roger, W. He, I. V. Yurkevich, and A. Kaplan, Appl. Phys. Lett. 101, 141904 (2012).

10. A. Kaplan, A. Sajwani, Z. Y. Li, R. E. Palmer and J. P. Wilcoxon, Appl. Phys. Lett. 88, 17105 (2006).

11. D. Chekulaev, V. Garber, and A. Kaplan, J. Appl. Phys 113, 143101 (2013).

12. R. A. Sinton and R. M. Swanson, IEEE Trans. Electron Devices ED-34, 1380 (1987).

13. J. Dziewior and W. Schmidt, Appl. Phys. Lett. 31, 346 (1977).

14. K. Svantesson and N. G. Nilsson, J. Phys. C 12, 5111 (1979).

15. I. A. Shkrob and R. A. Crowell, Phys. Rev. B 57, 12207 (1998).

16. J. Casado, M. A. Lopez-Quintela and F. M. Lorenzo-Barral, J. Chem. Educ. 63, 450 (1986).

17. A. Kaplan, A. Bekkerman, B. Tsipinyuk and E. Kolodney, J. Chem. Phys. 117, 3484 (2002).

18. A. Shah, J. Meier, E. Vallat-Sauvain, N. Wyrsch, U. Kroll, C. Droz, and U. Graf, Solar Energy Materials and Solar Cells 73, 469-491 (2003).

19. O. Vetterl, F. Finger, R. Carius, P. Hapke, L. Houben, O. Kluth, A. Lambertz, A. Mück, B. Rech, and H. Wagner, Solar Energy Materials and Solar Cells 62, 97-108 (2000).

20. P. Scherrer, Nachrichten von der Gesellschaft der Wissenschaften zu Göttingen, mathematisch-physikalische Klasse 1918, 98-100 (1918).

21. A. Patterson, Phys. Rev. 56, 978 (1939).

22. L. Houben, M. Luysberg, P. Hapke, R. Carius, F. Finger, and H. Wagner, Philosophical Magazine A 77, 1447-1460 (1998).

23. W. He, I. V. Yurkevich, A. Zakar, and A. Kaplan, Thin Solid Films pp. (2015).

24. M. C. Downer and C. V. Shank, Phys. Rev. Lett. 56, 761-764 (1986).

25. P. Findlay, C. Pidgeon, R. Kotitschke, A. Hollingworth, B. Murdin, C. Langerak, A. van Der Meer, C. Ciesla, J. Oswald, A. Homer et al., Physical Review B 58, 12908 (1998).

26. P. Jonsson, H. Bleichner, M. Isberg, and E. Nordlander, Journal of Applied Physics 81, 2256-2262 (1997).

27. A. W. Cohn, A. M. Schimpf, C. E. Gunthardt, and D. R. Gamelin, Nano Letters 13, 1810-1815 (2013).

28. J. P. Proot, C. Delerue and G. Allan, Appl. Phys. Lett. 61, 1948 (1992). 29. A. Schenk, Solid State Electronics 35, 1585 (1982).

30. A. Esser, K. Seibert, H. Kurz, G. Parsons, C. Wang, B. Davidson, G. Lucovsky, and R. Nemanich, Physical Review B 41, 2879 (1990). 
31. A. Esser, H. Heesel, H. Kurz, C. Wang, G. Parsons, and G. Lucovsky, Journal of Applied Physics 73, 1235-1239 (1993).

32. P. A. Wolff, Phys. Rev. 126, 405 (1962).

33. P. K. Basu, Theory of optical processes in semiconductors (Oxford University Press, 2003).

34. M. J. Powell and S. C. Deane, Phys. Rev. B 53, 10121-10132 (1996).

35. E. J. Yoffa, 2415 (1980).
1. R. A. Street, "Large area electronics, applications and requirements." Phys. Status Solidi A 166, 695-705 (1998).

2. A. Shah, P. Torres, R. Tscharner, N. Wyrsch, and H. Keppner, "Photovoltaic technology: the case for thin-film solar cells." Science 285, 692-698 (1999).

3. P. S. Yeh, C.-W. Chen, B.-R. Yang, and L.-S. Hong, "Lightscattering effectiveness of two-dimensional disordered surface textures in thin-film silicon solar cells," Appl. Opt. 53, 2847-2852 (2014).

4. S. Jung and J. Yi, "Nanocrystalline-silicon thin-film nonvolatile memory devices for display applications," Electron Device Letters, IEEE 31, 981-983 (2010).

5. C.-H. Lee, A. Sazonov, A. Nathan, and J. Robertson, "Directly deposited nanocrystalline silicon thin-film transistors with ultra high mobilities," Appl. Phys. Lett. 89, 252 (2006).

6. R. D. Kekatpure and M. L. Brongersma, "Fundamental photophysics and optical loss processes in si-nanocrystal-doped microdisk resonators," Phys. Rev. A 78, 023829 (2008).

7. K. E. Myers, Q. Wang, and S. L. Dexheimer, "Ultrafast carrier dynamics in nanocrystalline silicon," Phys. Rev. B 64, 161309 (2001).

8. J. Barreto, T. Roger, and A. Kaplan, "Resolving the ultrafast dynamics of charge carriers in nanocomposites," Appl. Phys. Lett. 100, 241906 (2012).

9. T. W. Roger, W. He, I. V. Yurkevich, and A. Kaplan, "Enhanced carrier-carrier interaction in optically pumped hydrogenated nanocrystalline silicon," Appl. Phys. Lett. 101, 141904 (2012).

10. A. Kaplan and A. Sajwani and Z. Y. Li and R. E. Palmer and J. P. Wilcoxon, "Efficient vacuum ultraviolet light frequency downconversion by thin films of CdSe quantum dots," Appl. Whys. Let. 88, 17105 (2006).

11. D. Chekulaev, V. Garber, and A. Kaplan, "Free carrier plasma optical response and dynamics in strongly pumped silicon nanopillars," J. Appl. Phys 113, 143101 (2013).

12. R. A. Sinton and R. M. Swanson, "Recombination in highly injected silicon," IEEE Trans. Electron Devices ED-34, 1380 (1987).

13. J. Dziewior and W. Schmidt, "Auger coefficients for highly doped and highly excited silicon," Appl. Phys. Lett. 31, 346 (1977).

14. K. Svantesson and N. G. Nilsson, "The temperature dependence of the auger recombination coefficient of undoped silicon," J. Phys. C 12, 5111 (1979).

15. I. A. Shkrob and R. A. Crowell, "Ultrafast charge recombination in undoped amorphous hydrogenated silicon," Physical Review B 57, 12207 (1998).

16. J. Casado , M. A. Lopez-Quintela and F. M. Lorenzo-Barral, J. Chem. Educ. 63, 450 (1986).

17. A. Kaplan, A. Bekkerman, B. Tsipinyuk and E. Kolodney, J. Chem. Phys. 117, 3484 (2002). 
18. A. Shah, J. Meier, E. Vallat-Sauvain, N. Wyrsch, U. Kroll, C. Droz, and U. Graf, "Material and solar cell research in microcrystalline silicon," Solar Energy Materials and Solar Cells 73, 469-491 (2003).

19. O. Vetterl, F. Finger, R. Carius, P. Hapke, L. Houben, O. Kluth, A. Lambertz, A. Mück, B. Rech, and H. Wagner, "Intrinsic microcrystalline silicon: A new material for photovoltaics," Solar Energy Materials and Solar Cells 62, 97-108 (2000).

20. P. Scherrer, "Bestimmung der grösse und der inneren struktur von kolloidteilchen mittels röntgenstrahlen," Nachrichten von der Gesellschaft der Wissenschaften zu Göttingen, mathematisch-physikalische Klasse 1918, 98-100 (1918).

21. A. Patterson, "The scherrer formula for $x$-ray particle size determination," Physical review 56, 978 (1939).

22. L. Houben, M. Luysberg, P. Hapke, R. Carius, F. Finger, and H. Wagner, "Structural properties of microcrystalline silicon in the transition from highly crystalline to amorphous growth," Philosophical Magazine A 77, 1447-1460 (1998).

23. W. He, I. V. Yurkevich, A. Zakar, and A. Kaplan, “Highfrequency conductivity of optically excited charge carriers in hydrogenated nanocrystalline silicon investigated by spectroscopic femtosecond pump-probe reflectivity measurements," Thin Solid Films pp. - (2015).

24. M. C. Downer and C. V. Shank, "Ultrafast heating of silicon on sapphire by femtosecond optical pulses," Phys. Rev. Lett. 56, 761-764 (1986).

25. P. Findlay, C. Pidgeon, R. Kotitschke, A. Hollingworth, B. Murdin, C. Langerak, A. van Der Meer, C. Ciesla, J. Oswald, A. Homer et al., "Auger recombination dynamics of lead salts under picosecond free-electron-laser excitation," Physical Review B 58, 12908 (1998).

26. P. Jonsson, H. Bleichner, M. Isberg, and E. Nordlander, "The ambipolar auger coefficient: Measured temperature dependence in electron irradiated and highly injected n-type silicon," Journal of applied physics 81, 2256-2262 (1997).

27. A. W. Cohn, A. M. Schimpf, C. E. Gunthardt, and D. R. Gamelin, "Size-dependent trap-assisted auger recombination in semiconductor nanocrystals," Nano letters 13, 18101815 (2013).

28. J. P. Proot, C. Delerue and G. Allan, Appl. Phys. Lett. 61, 1948 (1992).

29. A. Schenk, "A model for the field and temperature dependence of Shockley-Read-Hall Lifetimes in Silicon," Solid State Electronics 35, 1585 (1982).

30. A. Esser, K. Seibert, H. Kurz, G. Parsons, C. Wang, B. Davidson, G. Lucovsky, and R. Nemanich, "Ultrafast recombination and trapping in amorphous silicon," Physical Review B 41, 2879 (1990).

31. A. Esser, H. Heesel, H. Kurz, C. Wang, G. Parsons, and G. Lucovsky, "Femtosecond spectroscopic study of ultrafast carrier relaxation in hydrogenated amorphous silicon a-si: H," Journal of applied physics 73, 1235-1239 (1993).
32. P. A. Wolff, Phys. Rev. 126, 405 (1962).

33. P. K. Basu, Theory of optical processes in semiconductors (Oxford University Press, 2003).

34. M. J. Powell and S. C. Deane, "Defect-pool model and the hydrogen density of states in hydrogenated amorphous silicon," Phys. Rev. B 53, 10121-10132 (1996).

35. E. J. Yoffa, "Dynamics of dense laser-induced plasmas," Physical Review B 21, 2415 (1980). 